\title{
SOME STATISTICS OF SOUTHERN R CORONAE BOREALIS VARIABLES
}

\author{
VICKI E. SHER WOOD
}

Astronomisches Institut der Ruhr-Universität, Bochum, West Germany

\begin{abstract}
U B V$ observations of RCB stars with $\delta \leqslant 30^{\circ}$ were made at La Silla, Chile in the sunmer of 1972. Of 26 stars, 5 were below maximum brightness, including $\mathrm{R} \mathrm{CrB}$ and RY Sgr. On the basis of a comparison of the dispersion in observations of the RCB stars and nearby non-variable comparison stars, 9 RCB stars were found to show significant variation at maximum brightness. They are $\mathrm{W}$ Men, UX Ant, UW Cen, DY Cen, AE Cir, S Aps, RT Nor, RZ Nor, and V CrA. The amplitude of these variations ranges from 0.2 to 0.44 and tentative periods from 19 to 54 days and $>90$ days for S Aps. Similar variations at maximum are already known for R CrB, RY Sgr and XX Cam. Of the 5 stars which showed no significant variation at maximum, DZ And, Z Cir and V 973 Oph (see Orlov and Rodriguez, and Feast, this Symposium) are not RCB stars and SY Hyi is a questionable RCB star; only Y Mus is listed as a definite $R C B$ variable.

4 RCB stars in the Large Magellanic Cloud were observed: W Men, SY Hyi, HV 5637, and HV 12842. Absolute magnitudes at maximum of $-4 .^{m} 8,-6^{m} \cdot 6,-3^{m} \cdot 2$ and $-4^{m} \cdot 9$ respectively were derived. When plotted on a colour-colour diagram, most of these stars show an UV-excess for average colours at maximum. In the H-R diagram, they fall in a region where one would expect to find stars which are losing mass.
\end{abstract}

